\title{
Estimadores para atributos forestales en una población simulada de selva mediana del sur de México
}

\author{
Estimators for forest attributes on a simulated population \\ of medium-sized tropical forest in southern Mexico
}

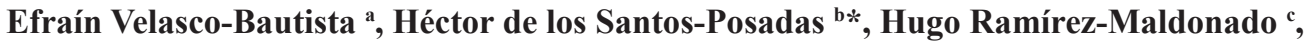 \\ Gilberto Rendón-Sánchez $^{\mathrm{d}}$, José René Valdez-Lazalde ${ }^{\mathrm{b}}$, Miguel Acosta-Mireles ${ }^{\mathrm{e}}$ \\ a Instituto Nacional de Investigaciones Forestales, Agrícolas y Pecuarias, \\ Centro Nacional de Investigación Disciplinaria en Conservación y Mejoramiento de Ecosistemas Forestales, \\ Ciudad de México, México. \\ *Autor de correspondencia: ${ }^{\text {b }}$ Colegio de Postgraduados, Postgrado en Ciencias Forestales, \\ Carretera México-Texcoco km 36.5, Montecillo, Estado de México, México, CP. 56230, tel.: 52-595-9520246, \\ hmsantos@colpos.mx \\ c Universidad Autónoma Chapingo, División de Ciencias Forestales. Chapingo, Estado de México. \\ ${ }^{\mathrm{d}}$ Colegio de Postgraduados, Postgrado en Socioeconomía, Estadística e Informática-Estadística, Montecillo, \\ Estado de México. \\ e Instituto Nacional de Investigaciones Forestales, Agrícolas y Pecuarias, Campo Experimental Valle de México, \\ Texcoco, Estado de México, México.
}

\begin{abstract}
SUMMARY
Throughout the data of the National Forest and Soil Inventory (INFyS) of Mexico, it is not uncommon to find clusters with less than four subplots (incomplete). The consequences of this condition on the forest parameters estimates are yet to be completely analyzed. The main objective of this work was to compare the behavior of different sampling estimators under such conditions of cluster completeness. Using an artificial population of 9,370,000 trees, created on a 10,000 ha surface, a total of 88 systematic sampling grids using four-plot circular clusters were set. Each grid had 81 clusters, separated by $1 \mathrm{~km}$. On each sampling grid, three different completeness conditions were tested: a) full completeness (all clusters with four subplots) b) $88 \%$ completeness and c) $63 \%$ completeness. On each condition, timber volume $\left(\mathrm{m}^{3} \mathrm{ha}^{-1}\right)$ and tree density (tree ha-1) were estimated using the following estimators: 1$)$ Forest Inventory and Analysis (FIA) 2) Van Deusen Estimators 3) Means of ratio and 4) Ratio of means. The estimators were evaluated using relative bias on the mean and the variance. For volume, on each of the three completeness conditions, the mean estimates were similar and unbiased using the proposed four estimators. Nevertheless, the FIA estimator produced biased variance estimates ranging from two to five times larger for $88 \%$ and $63 \%$ completeness respectively. Similar behavior was observed on tree density. The FIA estimators will produce biased results on the variance estimator when a high percentage of clusters is incomplete.
\end{abstract}

Key words: forest inventory, ratio of means, means of ratio, Van Deusen estimator, lognormal distribution.

\section{RESUMEN}

En el Inventario Nacional Forestal y de Suelos (INFyS) de México es frecuente obtener información en conglomerados menores a cuatro subparcelas. Las consecuencias de esto en las estimaciones de parámetros forestales no se han abordado lo suficiente. El objetivo del trabajo fue comparar cuatro estimadores para el volumen maderable y la densidad arbórea en tres condiciones de completitud de conglomerados. En una población artificial de 9.370 .000 árboles en 10.000 ha se diseñaron 88 redes sistemáticas de muestreo con conglomerados de subparcelas circulares; cada red estuvo conformada por 81 unidades de muestreo, equidistantes a $1 \mathrm{~km}$. En función del número de conglomerados completos se consideraron las siguientes condiciones de completitud: (a) al $100 \%$, (b) al $88 \%$ y (c) al $63 \%$. En cada condición se estimó el volumen maderable $\left(\mathrm{m}^{3} \mathrm{ha}^{-1}\right)$ y la densidad arbórea (árboles ha $\left.{ }^{-1}\right)$ mediante los estimadores: (1) Forest Inventory and Analysis (FIA), (2) Van Deusen (VAN), (3) Media de Razones (MR) y (4) Razón de Medias (RM). Para su comparación se usó el sesgo relativo, cuyos valores cercanos a cero son deseables. En cada condición analizada, las estimaciones de volumen por hectárea, para los cuatro estimadores, fueron similares e insesgadas. La varianza estimada mediante FIA fue sesgada en conglomerados incompletos, con valores superiores a dos y cinco veces la varianza verdadera para la segunda y tercera condición, respectivamente. Un comportamiento similar se observó para la densidad. Los estimadores FIA no son adecuados para estimar la varianza cuando se tiene un alto porcentaje de conglomerados incompletos.

Palabras clave: inventario forestal, razón de medias, media de razones, estimador Van Deusen, distribución log-normal. 


\section{INTRODUCCIÓN}

El diseño de muestreo del Inventario Nacional Forestal y de Suelos (INFyS) de México tiene como unidad de muestreo un conglomerado (parcela) integrado por cuatro subparcelas, las cuales están dispuestas geométricamente en forma de una " $Y$ " invertida con respecto al norte (CONAFOR 2012, CONAFOR 2018). Por razones diversas, existe la posibilidad que al utilizar el muestreo en campo algunos conglomerados tengan menos de cuatro subparcelas, lo cual conduce a conglomerados de tamaños distintos. Algunas de estas razones son: inaccesibilidad física a una parte del conglomerado, conglomerados físicamente truncados por orografía, cambios en el uso del suelo, estratificación o tipos de vegetación, entre otras. Dado que el porcentaje de conglomerados incompletos del INFyS de México, en algunos casos, es superior al $10 \%$ a nivel estatal, poco se ha explorado sobre cuáles son las consecuencias que el uso de conglomerados de distinto tamaño tiene en la precisión de las estimaciones (Van Deusen 2004, Velasco et al. 2005). Si existe variación notable entre los tamaños de los conglomerados y si estos están altamente correlacionados con los totales de los conglomerados, la varianza del total basada en la media muestral es generalmente mayor que la varianza basada en un estimador que considere el tamaño de los conglomerados como variable auxiliar (Lohr 2010, Scheaffer et al. 2012). Un ejemplo de esto es el estimador Razón de Medias (RM), que se recomienda cuando la varianza de la variable de interés es proporcional a la variable auxiliar.

Zarnoch y Bechtold (2000) señalan tres casos generales en los que el estimador de razón puede aplicarse para estimar parámetros forestales cuando se usan parcelas mapeadas, como unidades de muestreo, en el contexto del Forest Inventory Analysis Program (FIA) y el National Forest Health Monitoing Program (NFHM) de los Estados Unidos. Estos autores mencionan que los estimadores de razón pueden ser convenientemente aplicados en la estimación de: (a) atributos por hectárea, (b) atributos a nivel de árbol y (c) atributos a nivel de rodal. El estimador de razón, con el área efectivamente muestreada en el conglomerado como variable auxiliar, fue recomendado para la estimación de atributos forestales del Inventario Nacional Forestal de México (Velasco et al. 2005), el cual es un referente para la obtención de indicadores dasométricos a nivel nacional (CONAFOR 2018). Asimismo, los estimadores de razón de medias son recomendables incluso para la estimación de densidad arbolada en áreas urbanas (Álvarez et al. 2005) y para la estimación de atributos de salud a nivel de árbol, por ejemplo, la densidad de copa (Woodall et al. 2011, Bechtold y Randolph 2018). De manera alternativa el estimador de Media de Razones (MR) se recomienda cuando la varianza de la variable de interés es proporcional al cuadrado de la variable auxiliar (Scott et al. 2005).
De esta manera, Smelko y Merganic (2008) analizan los estimadores MR y RM en el contexto del Inventario y Monitoreo Forestal Nacional de Slovakia. Van Deusen (2004, 2005a) utiliza un estimador tipo RM, denominado estimador Van Deusen (VAN), pero expresan la varianza en términos del área de la parcela medida en campo. En estos casos, la variable de interés se correlaciona con el área de la parcela efectivamente muestreada en campo. En el contexto del FIA, Scott et al. (2005) y Van Deusen (2005a), estudian un estimador, llamado estimador Forest Inventory and Analysis (FIA), que toma en cuenta la proporción promedio de una parcela que está en la población de interés.

Al considerar que la cantidad del atributo de interés en el conglomerado es alguna función del área efectivamente muestreada del mismo y al tomar en cuenta los diferentes supuestos de la varianza del modelo lineal general que propone Van Deusen (2007), los estimadores FIA, VAN, MR y RM pueden derivarse empleando el método de mínimos cuadrados generalizados (Rencher y Bruce 2008). Dados los diferentes supuestos en los modelos, se espera que dichos estimadores y sus respectivas varianzas se desempeñen de manera distinta cuando se usen para estimar parámetros forestales agregados al emplear conglomerados incompletos.

Aun cuando un conglomerado del INFyS comprende dos o más subparcelas disjuntas, los datos que genera son analizados como una sola parcela debido a que el conglomerado es atado a un solo punto muestral. Así, en el análisis final, el conglomerado de subparcelas simplemente sirve para identificar aquellos elementos cuyas zonas de inclusión espacialmente disjuntas incluyen al punto muestral (Gregoire y Valentine 2008).

En este trabajo, los cuatro estimadores se analizan desde el contexto de conglomerados integrados por subparcelas disjuntas, no obstante, también pueden aplicarse cuando se tienen parcelas de área fija convencionales, siempre y cuando se cuantifique el área de la unidad de muestreo que corresponde a la población o estrato de interés.

En el presente estudio se utiliza una población artificial de 9.370 .000 árboles, construida con base en los parámetros de escala y de forma de una distribución lognormal ajustada con datos reales de la selva mediana subperennifolia de Quintana Roo, México, obtenidos del INFyS en 2010.

El objetivo del presente trabajo es comparar los estimadores FIA, VAN, MR y RM, al considerar el volumen maderable $\left(\mathrm{m}^{3} \mathrm{ha}^{-1}\right)$ y la densidad arbórea (árboles ha $\left.{ }^{-1}\right)$, en tres condiciones de completitud de los conglomerados. Para este fin, se utilizan como criterios el sesgo de la estimación relativo y el sesgo de la varianza relativo. La hipótesis de la investigación es que los cuatro estimadores puntuales y los estimadores de sus respectivas varianzas son insesgados independientemente del grado de completitud del conglomerado. 


\section{MÉTODOS}

Notación para derivar los estimadores estadísticos. Dado que en el presente estudio se evaluó el sesgo de la estimación puntual y el sesgo de la varianza de los estimadores FIA, VAN, MR y RM cuando se tienen parcelas de inventario en diferente grado de completitud es necesario presentar en detalle las respectivas estructuras matemáticas, las cuales pueden obtenerse mediante el modelo lineal general propuesto por Van Deusen (2007) empleando el método de mínimos cuadrados generalizados (Rencher y Bruce 2008). Para tal efecto, considérese la notación siguiente: $\mu$ : media de la variable $y$ por unidad de área (volumen, $\mathrm{m}^{3} \mathrm{ha}^{-1} \mathrm{y}$ densidad, árboles ha- $\left.{ }^{-1}\right) ; y_{s}:$ medición de la variable $y$ en la parcela $s ; a_{0}$ : área de la parcela $s$ ideal para medir la variable $y$, es decir, 0,16 ha (CONAFOR 2012, 2018); $a_{s}$ :área de la parcela $s$ (ha) efectivamente muestreada en campo; $m$ : número de parcelas que pertenecen a la población de interés.

Estimador FIA (Scott et al. 2005, Van Deusen 2005a). Al considerar una estimación de la varianza en forma convencional, la media y la varianza de FIA son, respectivamente, las siguientes:

$$
\begin{gathered}
\bar{y}_{h a}^{F L A}=\sum_{s=1}^{m} y_{h a}(s) / m \\
v\left(y_{h a}^{-F I A}\right)=\sum_{s=1}^{m}\left(y_{h a}(s)-\bar{y}_{h a}^{F I A}\right)^{2} / m(m-1)
\end{gathered}
$$

donde, $y_{\text {ha }}(s)=y_{s} / a_{0}\left(\sum_{s=1}^{m} a_{s} / a_{0} m\right)$

En la media de FIA $a_{0} m=\sum_{s=1}^{m} a_{0}$ es el área total desea ble a muestrear asumiendo conglomerados completos y $\sum_{s=1}^{m} a_{s}$ es el área total efectivamente muestreada. Entonces, $\sum_{s=1}^{m} a_{s} / a_{0} m \leq 1$. Así, la media de FIA considera el área del conglomerado completo ajustada por la proporción de las parcelas que pertenecen a la población de interés. Las expresiones de la media y varianza de FIA son los estimadores estándar de la media y la varianza que podrían aplicarse al muestreo aleatorio simple sin un factor de corrección por finitud (Cochran 1993 y Thompson 2002).

Estimador VAN (Van Deusen 2004, 2005a). Al usar en la estimación de la varianza el área de la parcela efectivamente muestreada en campo, se tienen para la media y la varianza de VAN los estimadores siguientes:

$$
y_{h a}{ }^{V A N}=\sum_{s=1}^{m} y_{s} / \sum_{s=1}^{m} a_{s}
$$

$$
v\left(y_{h a}^{V A N}\right)=\left[\sum_{s=1}^{m}\left(y_{s}-a_{s} y_{h a}\right)^{2}\right] /\left[\sum_{s=1}^{m} a_{s}\left(\sum_{s=1}^{m} a_{s}-a_{0}\right)\right]
$$

Estimador MR (Van Deusen 2005a). Al considerar una estimación de varianza convencional se obtienen los siguientes estimadores para la media y la varianza de MR:

$$
\begin{gathered}
\bar{y}_{h a}^{M R}=(1 / m) \sum_{s=1}^{m}\left(y_{s} / a_{s}\right) \\
v\left(\bar{y}_{h a}^{M R}\right)=\sum_{s=1}^{m}\left(y_{h a}-\bar{y}_{h a}^{M R}\right)^{2} / m(m-1)
\end{gathered}
$$

donde, $y_{h a}=y_{s} / a_{s}$

Estimador RM (Cochran 1993, Scheaffer et al. 2012). El estimador de razón tiene la misma estructura que el estimador VAN, el cual también puede ser usado cuando se presentan conglomerados incompletos, a saber:

$$
y_{h a}^{R M}=\sum_{s=1}^{m} y_{s} / \sum_{s=1}^{m} a_{s}
$$

La varianza estimada de este estimador es la siguiente:

$$
v\left(y_{h a}^{R M}\right)=\sum_{s=1}^{m}\left(y_{s}-y_{h a} a_{s}\right)^{2} / \bar{a}^{2} m(m-1)
$$

donde, $\bar{a}=\sum_{s=1}^{m} a_{s} / m$

Al considerar que $\bar{a}=\sum_{s=1}^{m} a_{s} / m \Rightarrow m=\sum_{s=1}^{m} a_{s} / \bar{a}$, el denominador de la varianza del estimador de razón puede expresarse en términos de las áreas efectivamente muestreadas, lo que conduce a la siguiente expresión:

$$
v\left(y_{h a}^{R M}\right)=\sum_{s=1}^{m}\left(y_{s}-y_{h a} a_{s}\right)^{2} /\left(\sum_{s=1}^{m} a_{s}\left(\sum_{s=1}^{m} a_{s}-\bar{a}\right)\right)
$$

Consideraciones generales sobre los estimadores. Una inspección detallada de la expresión (4) y (9) permite apreciar que ambas solo difieren en el denominador por el término que se sustrae del total de área efectivamente muestreada, mientras que la varianza del estimador de razón considera un promedio, en VAN se resta el área de un conglomerado completo, es decir, 0,16 ha. Si todos los conglomerados de la muestra son completos, se tiene que $\bar{a}=\sum_{s=1}^{m} a_{0} / m=a_{0} m / m=a_{0}, \mathrm{y}$, en consecuencia, la varianza del estimador de razón y Van Deusen conducen prácticamente a los mismos resultados. De hecho, $a_{0}=0,16$ es el máximo valor que se puede considerar, luego entonces, $a \leq a_{0}$. En estas condiciones la varianza estimada del estimador de razón siempre será ligeramente más pequeña que Van Deusen. 
La varianza del estimador de razón puede obtenerse multiplicando la varianza del estimador VAN por un factor que involucra $\bar{a}$, es decir, $v\left(y_{h a}^{R M}\right)=\left(\left(m \bar{a}-a_{0}\right) /(\bar{a}(m-1))\right) v\left(y_{h a}^{V A N}\right)$. En esta expresión nuevamente se observa que cuando $\bar{a}=a_{0}$, $v\left(y_{h a}^{R M}\right)=v\left(y_{h a}^{V A N}\right)$. Una situación extrema ocurre cuando $\bar{a}=(1 / 2) a_{0}$ y $m=20$, resultando que el cociente de la varianza del estimador de razón respecto a la varianza del estimador de Van Deusen es de 0,9473 (Van Deusen 2005b).

Cuando los conglomerados están completos, es decir, $a_{s}=a_{0}$, los cuatro estimadores coinciden, $\mathrm{y}$

$$
y_{h a}=\sum_{s=1}^{m} y_{s} / a_{0} m
$$

además, los cuatro estimadores presentan las mismas varianzas estimadas, y conduce a la expresión siguiente:

$$
v\left(y_{h a}\right)=\left(\sum_{s=1}^{m}\left(y_{s}-\sum_{s=1}^{m} y_{s} / m\right)^{2}\right) /\left(a_{0}^{2} m(m-1)\right)[11]
$$

Con base en estas afirmaciones queda de manifiesto que la estimación por hectárea cuando los conglomerados son completos se obtiene promediando valores por conglomerado y el resultado se expande por hectárea mediante $1 / a_{0}$. Este factor de expansión, al ser una constante, también aparece en la varianza. Esta situación es de particular importancia porque las fórmulas se reducen a los estimadores del muestreo aleatorio simple.

Base de datos artificial considerando información del INFyS. La metodología utilizada en esta investigación fue similar a la empleada por Condes y Martínez-Millán (1998), quienes simularon parcelas al considerar como base los sitios del segundo inventario forestal nacional de España en lo que se refiere al número de árboles por hectárea y a la distribución diamétrica de los árboles; y Stamatellos y Panourgias (2005), quienes utilizaron una muestra aleatoria de 229 parcelas de 0,05 hectárea $(20 \times 25 \mathrm{~m})$ para simular la distribución espacial de Abies borisii regis en la Universidad Forestal de Pertouli, Trikala, Grecia.

$\mathrm{Al}$ asumir que los árboles en una selva mediana subperennifolia presentan un patrón espacial aleatorio $\mathrm{y}$, por lo tanto, la misma probabilidad de encontrarse en cualquier lugar del terreno, en el sistema de análisis estadístico SAS ${ }^{\circledR}$ v9.2 (SAS Institute Inc. 2009) se generó una población aleatoria de 9.370 .000 puntos en la parte central de Quintana Roo, México. Cada uno de estos puntos representó un árbol. Los números en el eje x se obtuvieron mediante el código Int((MaxValue-MinValue +1) *ranuni) + MinValue, en donde, MaxValue y MinValue correspondieron a los valores extremos en $\mathrm{x}(\mathrm{m})$ de la región de estudio, y ranuni, sirvió para generar datos con distribución uniforme. Un código similar se aplicó para generar los valores en el eje y. La posición geográfica de los puntos se definió mediante las coordenadas (x, y). Los valores extremos de $\mathrm{x}$ fueron $3.894 .331,69 \mathrm{~m}$ y $3.904 .331,69 \mathrm{~m}$ y de $\mathrm{y}$ fueron 889.927,12 m y 899.927,12 m (proyección conforme cónica de Lambert, Datum ITRF92, elipsoide GRS80), los cuales se definieron de manera que el área de estudio representara 10.000 ha y la densidad promedio fuera 937 árboles ha-1.

El volumen de cada árbol se generó a través de una distribución probabilística log-normal ajustada con datos reales en la región de estudio mediante la expresión siguiente:

VOLUMEN $=\operatorname{EXP}(-2,67507+0,906635 * \operatorname{RANNOR}(100))$

donde,

2,67507= parámetro de escala de la distribución log-normal.

$0,906635=$ parámetro de forma de la distribución log normal.

$\mathrm{EXP}=$ función exponencial.

RANNOR (100) = función generadora de números aleatorios normales con semilla de 100 .

El cálculo del número de árboles por hectárea y el ajuste de la distribución log-normal se realizó con datos del INFyS obtenidos en 2010 en selva mediana subperennifolia de Quintana Roo, México. Para esto se utilizó un tamaño de muestra de 206 conglomerados, de los cuales 25 estuvieron incompletos: cinco presentaron un sitio, siete tenían dos sitios y 13 contaron con tres sitios; lo que representa un $12 \%$ de conglomerados incompletos.

Muestreo en redes sistemáticas. En SAS ${ }^{\circledR}$ v9.2 se diseñaron 88 redes sistemáticas diferentes (repeticiones), cada una de 81 puntos de muestreo, los cuales estuvieron a equidistancias de $1 \mathrm{~km}$; cada punto representó el centro (subparcela número 1) de un conglomerado de subparcelas (figura 1). Este tamaño de muestra justifica el uso de la distribución normal en los intervalos de confianza para la media y es similar al usado por Van Deusen (2004, 2005a). Mediante las funciones trigonométricas seno y coseno se generaron los centros de las unidades de muestreo periféricas del conglomerado (subparcelas 2, 3 y 4). En cada malla de muestreo se generaron conglomerados con subparcelas circulares.

Las coordenadas de puntos aleatorios (árboles) y de centros correspondientes a las cuatro subparcelas de conglomerados se capturaron en el sistema de información geográfica $\operatorname{ArcGis}{ }^{\circledR}$ v9.3 (ESRI, 2008). Las subparcelas circulares se generaron a través de la herramienta "buffer" de ArcToolbox-ArcMap considerando una distancia de 11,28 m. Los conglomerados trazados fueron similares al diseño de la unidad de muestreo del INFyS de México (figura 1). El muestreo de los puntos (árboles) utilizando 
conglomerados de subparcelas circulares dispuestas espacialmente en forma de una "Y" invertida, se realizó mediante la herramienta "Intersect" de ArcToolbox-ArcMap.

De los 81 conglomerados completos se modificaron diez de ellos con el fin de simular conglomerados incompletos; esto se realizó de manera proporcional al número de conglomerados con menos de cuatro sitios que presentó la muestra de 206 conglomerados, misma que sirvió de base para simular la población de 9.370 .000 puntos distribuidos aleatoriamente en el terreno. Los diez conglomerados incompletos se integraron de la manera siguiente: dos con una subparcela, tres con dos sub-parcelas y cinco con tres sub-parcelas. Tomando como base esta condición y triplicando el número de conglomerados con una, dos y tres subparcelas, adicionalmente se generaron 30 conglomerados incompletos. Entonces, el estudio se llevó a cabo en las condiciones siguientes: completitud total ( $\mathrm{c} 0)$ incluye 81 conglomerados completos, completitud al $88 \%$ (c1) comprende 71 conglomerados completos y diez incompletos (dos con una subparcela, tres con dos sub-parcelas y cinco con tres sub-parcelas) y completitud al $63 \%$ (c2) considera 51 conglomerados completos y 30 incompletos (seis con una sub-parcela, nueve con dos sub-parcelas y 15 con tres sub-parcelas).

Comparación de estimadores. Los cuatro estimadores estudiados en cada condición se compararon mediante el sesgo de estimación relativo (SER) y el sesgo de varianza relativo (SVR), cuyas expresiones genéricas, de acuerdo con Van Deusen (2005a), son las siguientes:

$$
S E R=(\hat{\bar{\theta}}-\theta) / \theta
$$

$$
S V R=(\hat{\bar{V}}-V) / V
$$

donde,

$\hat{\bar{\theta}}=\sum_{b=1}^{88} \hat{\theta}_{b} / 88$, en cada caso $\hat{\theta}_{b}$ se obtiene de acuerdo con las expresiones [1], [3], [5] y [7].

$\theta=937$ es el parámetro de densidad (árboles ha ${ }^{-1}$ )

$\theta=97,37$ es el parámetro de volumen $\left(\mathrm{m}^{3} \mathrm{ha}^{-1}\right)$

$V=\sum_{b=1}^{88}\left(\hat{\theta}_{b}-\hat{\bar{\theta}}\right)^{2} / 88$ es la varianza verdadera

$\hat{\bar{V}}=\sum_{b=1}^{88} \hat{V}_{b} / 88$, en cada caso $\hat{V}_{b}$ se obtiene de acuerdo con las expresiones [2], [4], [6] y [9].

De acuerdo con Van Deusen (2005a), en las expresiones del sesgo relativo, un valor cercano a 0 significa que el estimador es insesgado, un valor de 1 muestra que la fórmula predice dos veces al parámetro y un valor de 2 indica que la fórmula sobrestima por factor de 3. En el presente estudio, la condición de insesgadez implica sesgos relativos cercanos cero; de esta manera, un estimador se consideró insesgado si su sesgo relativo fue menor al 0,10 . En este sentido, el sesgo relativo carece de unidades y se interpreta como un indicador del número de veces que el estimador rebasa o contiene al parámetro o valor verdadero.

Figura 1. Distribución sistemática de los conglomerados y disposición geométrica de las subparcelas (círculos pequeños de $11,84 \mathrm{~m}$ de radio) dentro del conglomerado (círculo grande de 56,42 $\mathrm{m}$ de radio). $m$ radius).

Systematic grid of clusters and geometric array of the sub-plots (small circles of 11.84 m radius) within each cluster (larger circle of 56.42 


\section{RESULTADOS}

Volumen maderable. En la población simulada de árboles, el parámetro correspondiente al volumen fue de $97,37 \mathrm{~m}^{3}$ $\mathrm{ha}^{-1}$. En c0 (nivel de completitud total), como era de esperarse, los cuatro estimadores presentaron el mismo valor puntual: para las 88 repeticiones estimaron en promedio un volumen de $97,76 \mathrm{~m}^{3} \mathrm{ha}^{-1}$ (figura 2) y, en consecuencia, el sesgo de la estimación relativo fue prácticamente cero, es decir, 0,004 . Así, en c0 y para el volumen, los cuatro estimadores se consideran insesgados. En los cuatro casos, la varianza promedio estimada, a partir de las 88 repeticiones, fue de $1,79\left(\mathrm{~m}^{3} \mathrm{ha}^{-1}\right)^{2}$ (figura 3 ) y la varianza verdadera de $1,82\left(\mathrm{~m}^{3} \mathrm{ha}^{-1}\right)^{2}$, lo cual condujo a un sesgo de la varianza relativo muy cercano a cero, o sea, $-0,0165$. De esta manera, en c0 y para el volumen maderable, los cuatros estimadores de la varianza también resultan insesgados.

Estos resultados son optimistas en el sentido de que cuando en los conglomerados se toma la información en las cuatro subparcelas, cualquiera de los estimadores evaluados en este trabajo pueden ser de utilidad.

En c1 (nivel de completitud al $88 \%$ ), los estimadores RM, FIA y VAN coincidieron y estimaron en promedio, a partir de las 88 repeticiones, un volumen de $97,69 \mathrm{~m}^{3} \mathrm{ha}^{-1}$, el cual resultó ser ligeramente diferente de la estimación de MR, cuyo valor fue de $97,60 \mathrm{~m}^{3} \mathrm{ha}^{-1}$ (figura 4). En el primer caso, el sesgo de la estimación relativo fue de 0,0033 , y en el segundo, de 0,0024. Entonces, en la condición c1, al presentar sesgos próximos a cero, los cuatro estimadores del volumen se consideran prácticamente insesgados. Por otra parte, mientras que la varianza verdadera de RM, FIA y VAN fue de 2,04 $\left(\mathrm{m}^{3} \mathrm{ha}^{-1}\right)^{2}$ y la de MR de 2,22 $\left(\mathrm{m}^{3} \mathrm{ha}^{-1}\right)^{2}$, la varianza estimada de FIA ascendió hasta un promedio de $5,18\left(\mathrm{~m}^{3} \mathrm{ha}^{-1}\right)^{2}$, en tanto que la varianza promedio estimada de RM y VAN fue de $1,88\left(\mathrm{~m}^{3} \mathrm{ha}^{-1}\right)^{2}$ y la de MR de 2,03 $\left(\mathrm{m}^{3}\right.$ $\left.\mathrm{ha}^{-1}\right)^{2}$ (figura 5). De esta forma, el sesgo de la varianza relativo para RM y VAN fue de $-0,0784$, para MR de $-0,0856$ y para FIA ascendió hasta 1,5392. En consecuencia, en c1 la varianza estimada para MR, RM y VAN también se considera insesgada cuando se estima el volumen maderable. Sin embargo, la varianza estimada de FIA, al ser más de dos veces la varianza verdadera, se considera sesgada.

En c2 (nivel de completitud al $63 \%$ ), los estimadores RM, FIA y VAN coincidieron y en promedio estimaron $97,61 \mathrm{~m}^{3} \mathrm{ha}^{-1}$; variaron poco con MR, el cual estimó 97,65 $\mathrm{m}^{3} \mathrm{ha}^{-1}$ (figura 6). De esta manera, el sesgo de la estimación relativo correspondiente a RM, FIA y VAN fue de 0,0025 , mientras que para MR fue de 0,0029. Entonces, aún en el nivel de completitud al $63 \%$, los cuatro estimadores resultaron con sesgos relativos pequeños y, por consiguiente, se consideran insesgados. En tanto que la varianza verdadera de RM, FIA y VAN fue de 2,19 $\left(\mathrm{m}^{3}\right.$ $\left.\mathrm{ha}^{-1}\right)^{2}$ y la de MR de 2,69 (m $\mathrm{m}^{3}$ ha-1 $)^{2}$, la varianza estimada de FIA alcanzó en promedio $11,51\left(\mathrm{~m}^{3} \mathrm{ha}^{-1}\right)^{2}$, superando por mucho la varianza promedio estimada de RM y VAN que fue de 2,12 $\left(\mathrm{m}^{3} \mathrm{ha}^{-1}\right)^{2}$ y la de MR que fue de 2,50 $\left(\mathrm{m}^{3}\right.$ $\left.\mathrm{ha}^{-1}\right)^{2}$ (figura 7). Entonces, el sesgo de la varianza relativo correspondiente a RM y VAN fue de $-0,0320$, para MR de $-0,0706$ y para FIA alcanzó un valor de 4,2557 . De manera que en c2, los estimadores de la varianza para MR, RM y VAN también son insesgados, porque en ningún caso el sesgo de la varianza relativo superó el 0,10 . No obstante,

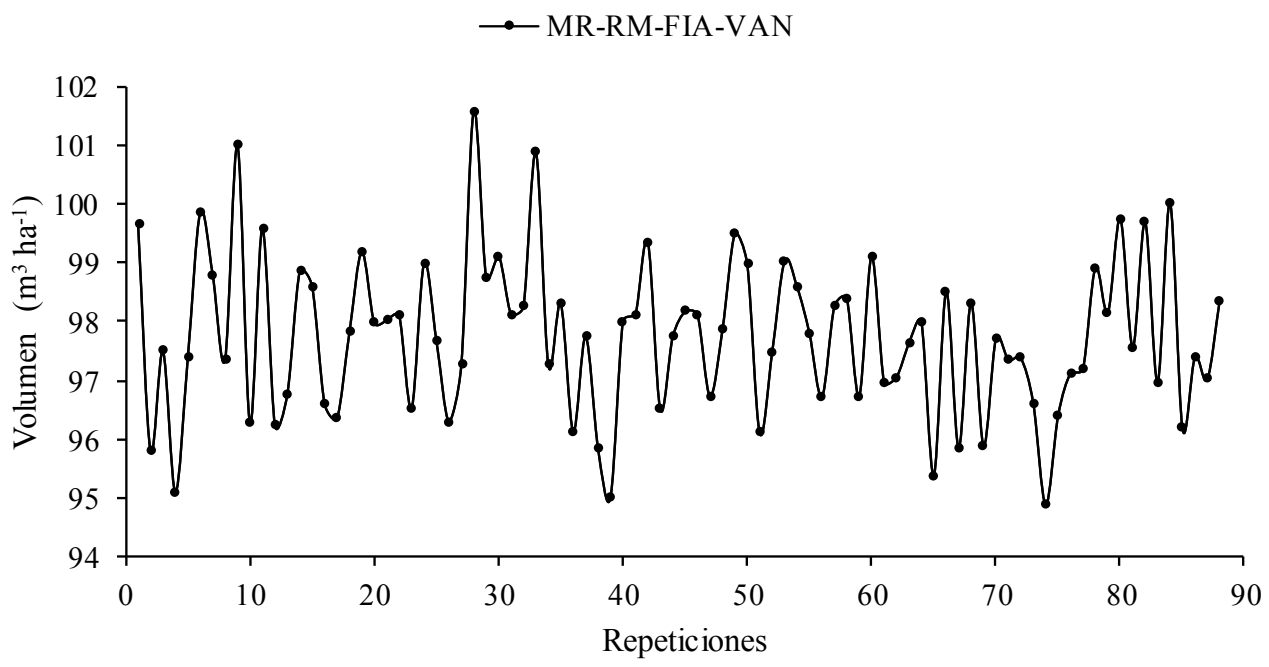

Figura 2. Volumen maderable estimado $\left(\mathrm{m}^{3} \mathrm{ha}^{-1}\right)$ mediante cuatro estimadores estudiados en la condición c0. FIA: Forest Inventory and Analysis, VAN: Van Deusen, MR: Media de Razones, RM: Razón de Medias.

Estimated timber volume $\left(\mathrm{m}^{3} \mathrm{ha}^{-1}\right)$ of the four studied estimators on the $\mathrm{c} 0$ condition. FIA: Forest Inventory and Analysis, VAN: Van Deusen, MR: Means of ratio, RM: Ratio of means. 
la varianza estimada de FIA en la condición c2, al superar por más de cinco veces la varianza verdadera, puede considerarse altamente sesgada.

De acuerdo con los resultados obtenidos, los cuatros estimadores estudiados para la media del volumen maderable por hectárea presentaron un comportamiento insesgado independientemente del grado de completitud del conglomerado. No obstante, el sesgo de la varianza relati- vo del FIA se incrementó considerablemente conforme el grado de completitud disminuyó.

Densidad arbórea. En la población, la densidad promedio fue de 937 árboles ha-1 ${ }^{-1}$ Resulta interesante observar nuevamente que en la condición c0 los cuatros estimadores condujeron a los mismos resultados: 939,94 árboles ha-1 promedio de las 88 repeticiones (figura 8 ), lo que resul-

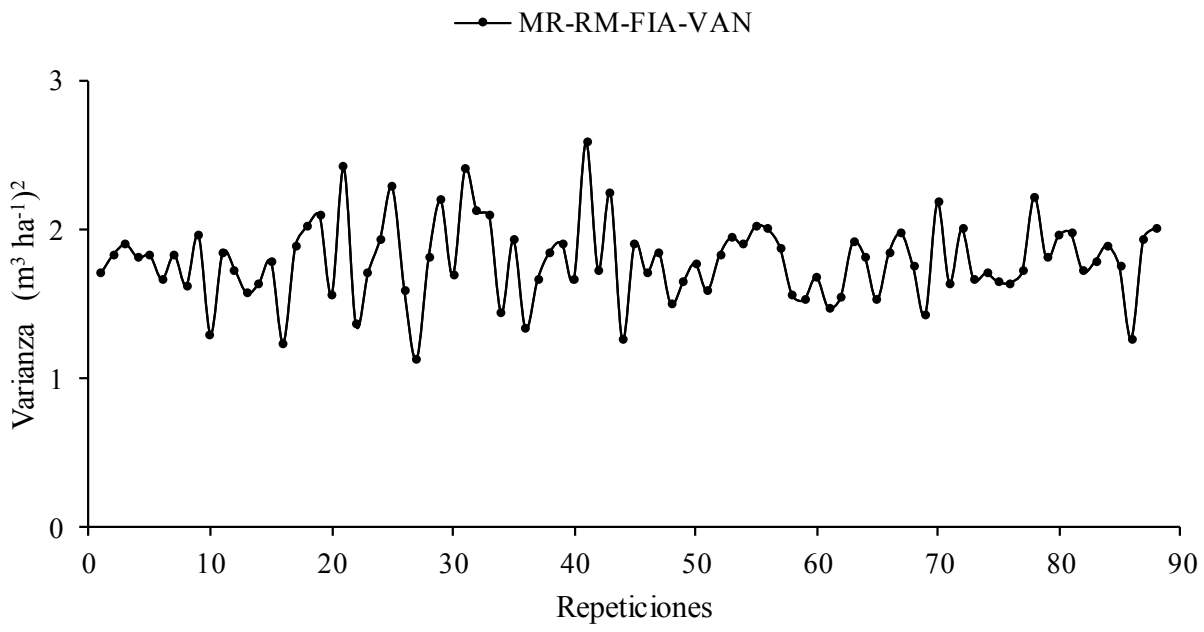

Figura 3. Varianza estimada $\left(\mathrm{m}^{3} \mathrm{ha}^{-1}\right)^{2}$ de cuatro estimadores para volumen maderable estudiados en la condición c0. FIA: Forest Inventory and Analysis, VAN: Van Deusen, MR: Media de Razones, RM: Razón de Medias.

Estimated timber volume variance $\left(\mathrm{m}^{3} \mathrm{ha}^{-1}\right)^{2}$ of the four studied estimators on the $\mathrm{c} 0$ condition. FIA: Forest Inventory and Analysis, VAN: Van Deusen, MR: Means of ratio, RM: Ratio of means.

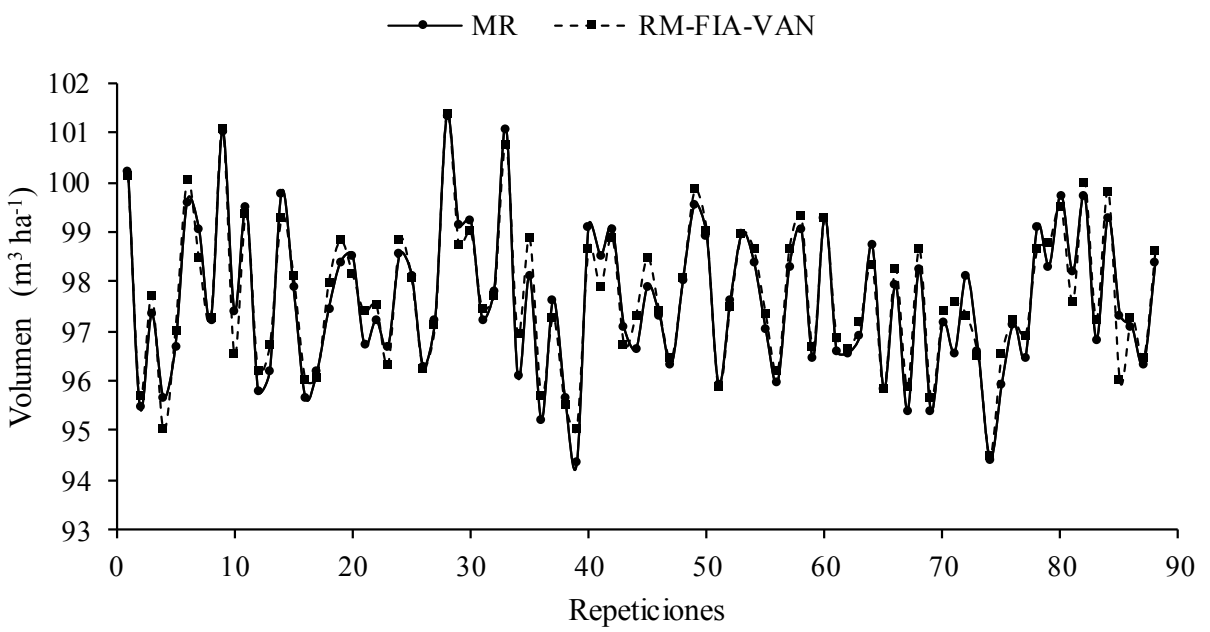

Figura 4. Volumen maderable estimado $\left(\mathrm{m}^{3} \mathrm{ha}^{-1}\right)$ mediante cuatro estimadores estudiados en la condición c1. FIA: Forest Inventory and Analysis, VAN: Van Deusen, MR: Media de Razones, RM: Razón de Medias.

Estimated timber volume $\left(\mathrm{m}^{3} \mathrm{ha}^{-1}\right)$ of the four studied estimators on the $\mathrm{c} 1$ condition. FIA: Forest Inventory and Analysis, VAN: Van Deusen, MR: Means of ratio, RM: Ratio of means. 
tó en un sesgo de la estimación relativo de 0,0031. Para los cuatro estimadores se obtuvo una varianza promedio estimada de 72,99 (árboles ha-1)2 (figura 9), la cual prácticamente fue la misma que la varianza verdadera, 72,92 $\left(\text { árboles } \mathrm{ha}^{-1}\right)^{2}$, lo que derivó en un sesgo de la varianza relativo de 0,0010 . Dada la magnitud de los sesgos relativos, en $\mathrm{c} 0$ y para la variable densidad, se deduce que tanto los estimadores como sus respectivas varianzas estimadas son insesgados.

En c1, mediante RM, FIA y VAN se obtuvo una densidad promedio de 939,48 árboles ha ${ }^{-1}$, mismo que superó ligeramente a la estimación de MR, cuyo valor fue de 939,26 árboles ha-1 (figura 10); así, al considerar el parámetro, los sesgos de la estimación relativos fueron de 0,0026 y

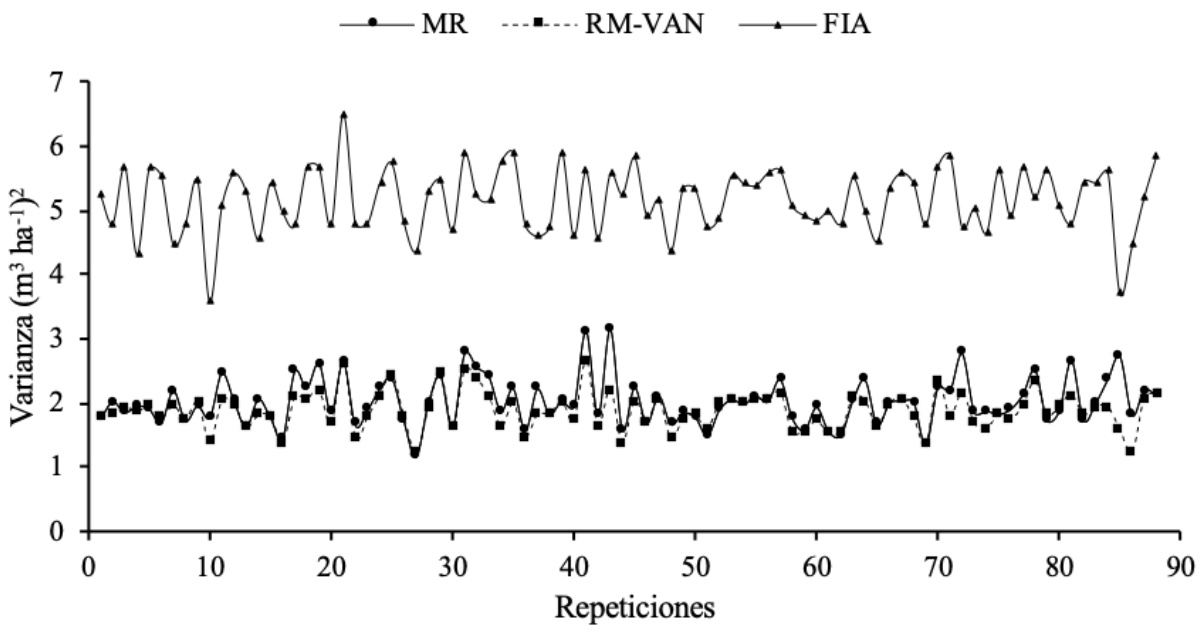

Figura 5. Varianza estimada $\left(\mathrm{m}^{3} \mathrm{ha}^{-1}\right)^{2}$ de cuatro estimadores para volumen maderable estudiados en la condición c1. FIA: Forest Inventory and Analysis, VAN: Van Deusen, MR: Media de Razones, RM: Razón de Medias.

Estimated timber volume variance $\left(\mathrm{m}^{3} \mathrm{ha}^{-1}\right)^{2}$ of the four studied estimators on the $\mathrm{c} 1$ condition. FIA: Forest Inventory and Analysis, VAN: Van Deusen, MR: Means of ratio, RM: Ratio of means.

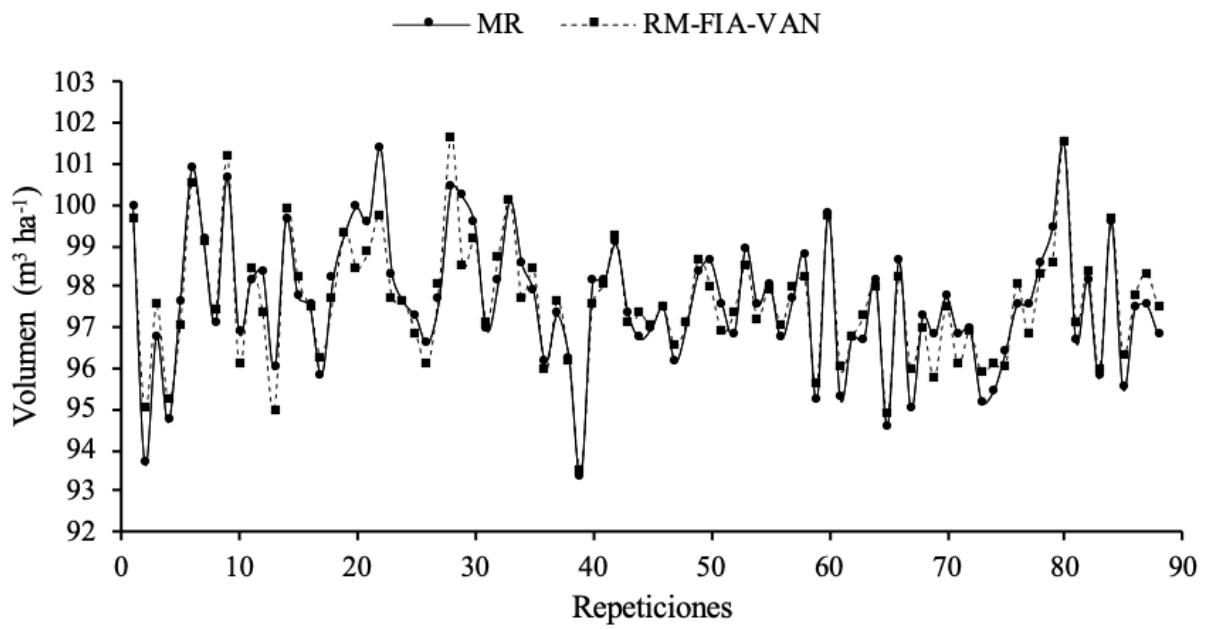

Figura 6. Volumen maderable estimado $\left(\mathrm{m}^{3} \mathrm{ha}^{-1}\right)$ mediante cuatro estimadores estudiados en la condición c2. FIA: Forest Inventory and Analysis, VAN: Van Deusen, MR: Media de Razones, RM: Razón de Medias.

Estimated timber volume $\left(\mathrm{m}^{3} \mathrm{ha}^{-1}\right)$ of the four studied estimators on the c2 condition. FIA: Forest Inventory and Analysis, VAN: Van Deusen, MR: Means of ratio, RM: Ratio of means. 
0,0024, respectivamente. De esta manera, los cuatro estimadores resultan ser insesgados. Por otro lado, en tanto la varianza verdadera correspondiente a RM, FIA y VAN fue de 80,44 (árboles ha $\left.{ }^{-1}\right)^{2}$ y MR de 83,62 (árboles ha-1)2 ${ }^{2}$, la varianza estimada promedio de FIA ascendió hasta 375,50 $\left(\right.$ árboles ha-1) ${ }^{2}$, la de RM y VAN fue 76,08 (árboles ha-1)2 y la de MR de 82,39 (árboles ha-1)2 (figura 11). Si bien, las varianzas estimadas de RM, VAN y MR son insesgadas, la varianza estimada de FIA se considera sesgada, al superar la varianza verdadera por más de cuatro veces.

En c2, RM, FIA y VAN estimaron una densidad prome-

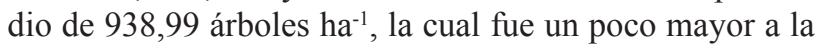
estimación de MR, cuyo promedio fue de 938,59 árboles ha $^{-1}$ (figura 12); de este modo, los sesgos de la estimación

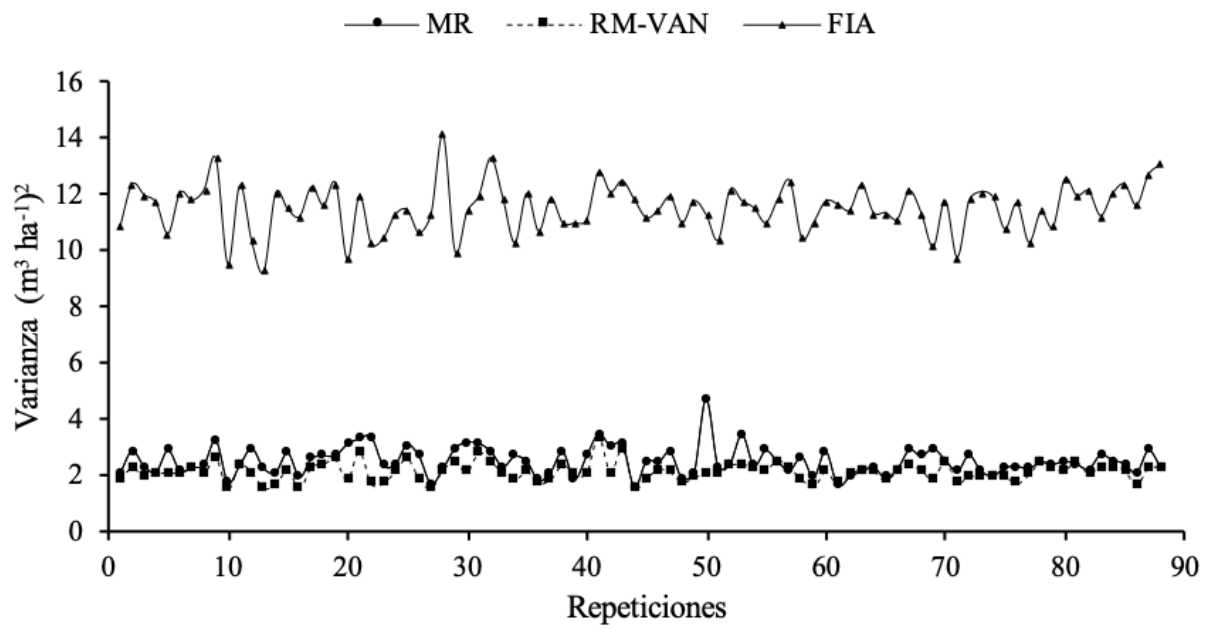

Figura 7. Varianza estimada $\left(\mathrm{m}^{3} \mathrm{ha}^{-1}\right)^{2}$ de cuatro estimadores para volumen maderable estudiados en la condición c2. FIA: Forest Inventory and Analysis, VAN: Van Deusen, MR: Media de Razones, RM: Razón de Medias.

Estimated timber volume variance $\left(\mathrm{m}^{3} \mathrm{ha}^{-1}\right)^{2}$ of the four studied estimators on the $\mathrm{c} 2$ condition. FIA: Forest Inventory and Analysis, VAN: Van Deusen, MR: Means of ratio, RM: Ratio of means.

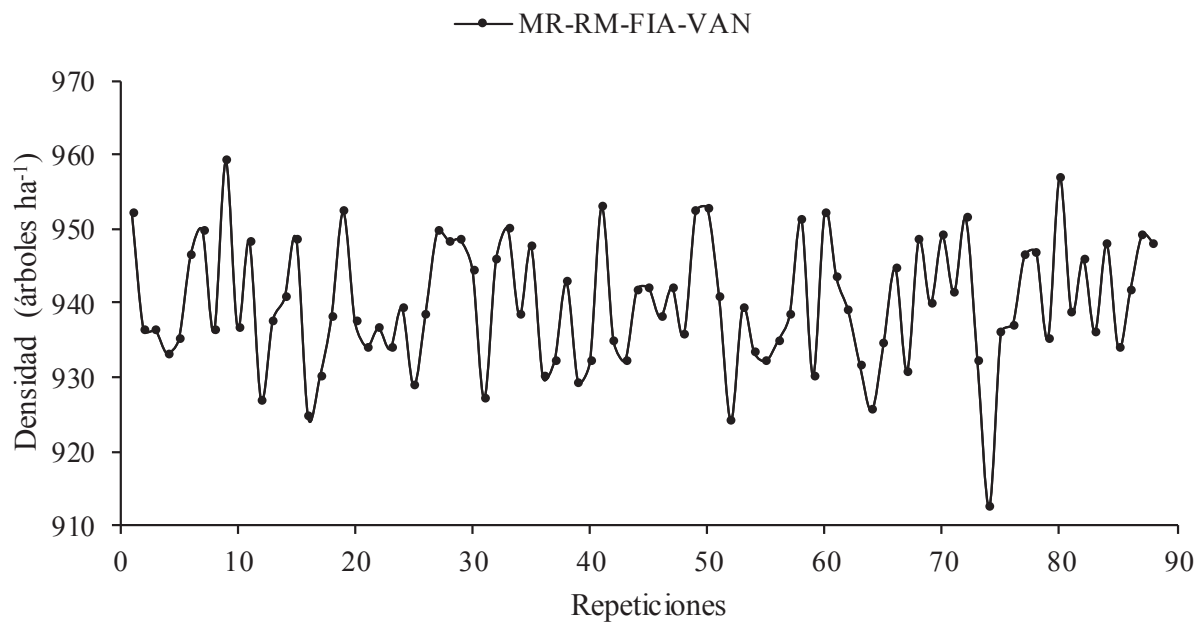

Figura 8. Densidad estimada (árboles ha ${ }^{-1}$ ) mediante cuatro estimadores estudiados en la condición c0. FIA: Forest Inventory and Analysis, VAN: Van Deusen, MR: Media de Razones, RM: Razón de Medias.

Estimated tree density (trees $\mathrm{ha}^{-1}$ ) using the four studied estimators on the $\mathrm{c} 0$ condition. FIA: Forest Inventory and Analysis, VAN: Van Deusen, MR: Means of ratio, RM: Ratio of means. 
relativos fueron respectivamente de 0,0021 y 0,0017 . De esta manera, en c2 los cuatro estimadores pueden considerarse insesgados. Por otra parte, mientras la varianza verdadera correspondiente a RM, FIA y VAN fue de 84,80 $\left(\text { árboles ha }{ }^{-1}\right)^{2}$ y MR de 96,51 (árboles ha $\left.{ }^{-1}\right)^{2}$, la varianza estimada promedio de FIA logró la cantidad de 965,45 (árboles $\left.\mathrm{ha}^{-1}\right)^{2}$, la de RM y VAN fue 87,30 (árboles ha-1 h $^{2}$ y la de MR de 102,24 (árboles ha-1 ${ }^{2}$ (figura 13). En tanto que las varianzas estimadas de RM, VAN y MR presentaron sesgos relativos cercanos a cero y se consideran insesgadas, la varianza estimada de FIA resulta altamente sesgada, al superar la varianza verdadera por más de once veces.

\section{DISCUSIÓN}

Los resultados obtenidos en la condición c0, sobre estimaciones y varianzas estimadas de volumen maderable y densidad considerando los cuatro estimadores, son idén-

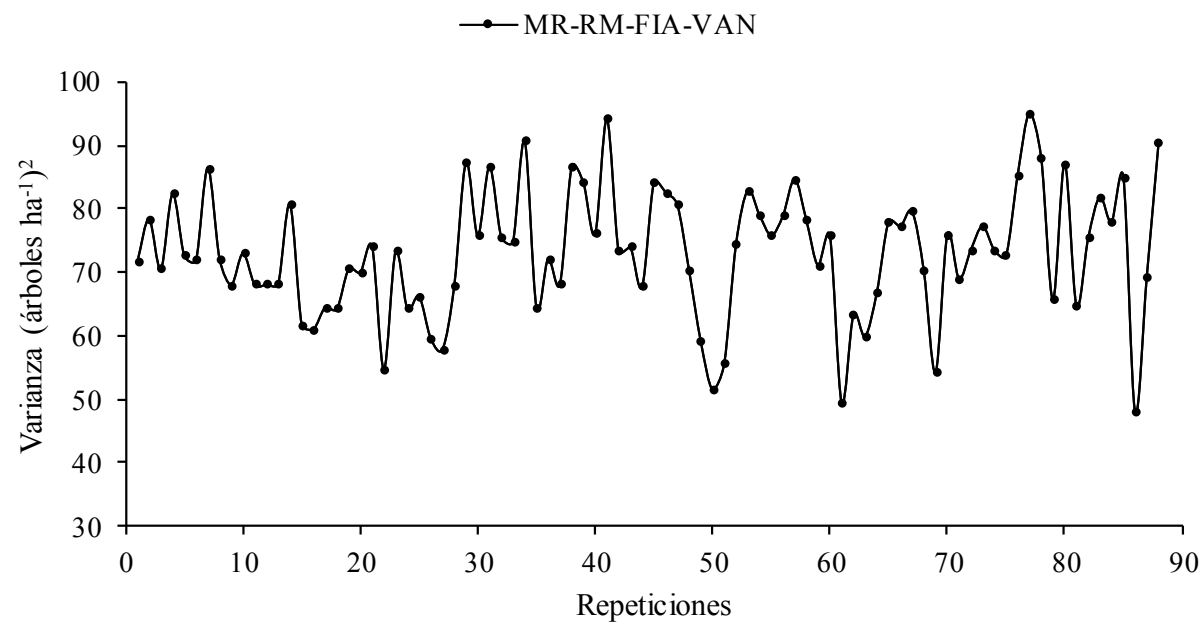

Figura 9. Varianza estimada (árboles ha $\left.\mathrm{a}^{-1}\right)^{2}$ de cuatro estimadores estudiados en la condición c0. FIA: Forest Inventory and Analysis, VAN: Van Deusen, MR: Media de Razones, RM: Razón de Medias.

Estimated variance $\left(\text { trees } \mathrm{ha}^{-1}\right)^{2}$ on the four studied estimators on the $\mathrm{c} 0$ condition. FIA: Forest Inventory and Analysis, VAN: Van Deusen, MR: Means of ratio, RM: Ratio of means.

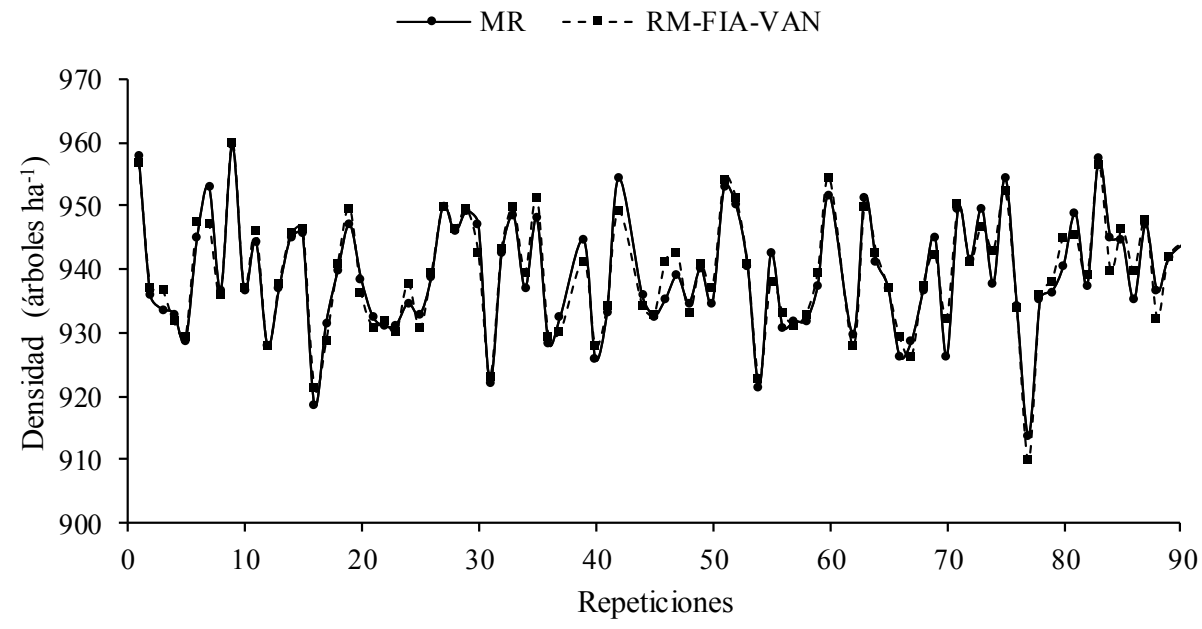

Figura 10. Densidad estimada (árboles ha ${ }^{-1}$ ) mediante cuatro estimadores estudiados en la condición c1. FIA: Forest Inventory and Analysis, VAN: Van Deusen, MR: Media de Razones, RM: Razón de Medias.

Estimated tree density (trees $\mathrm{ha}^{-1}$ ) using the four studied estimators on the $\mathrm{c} 1$ condition. FIA: Forest Inventory and Analysis, VAN: Van Deusen, MR: Means of ratio, RM: Ratio of means. 
ticos; lo cual era de esperarse, debido a que cuando los conglomerados son del mismo tamaño se tiene un estimador común. Así, bajo c0 (nivel de completitud total) puede aplicarse cualquiera de los cuatro estimadores ensayados en este trabajo, inclusive el estimador FIA, cuya expresión matemática considera una proporción promedio de la parcela que pertenece a la población de interés. Esta situación es de particular importancia porque las fórmulas se reducen a los estimadores convencionales del muestreo aleatorio simple. Por otra parte, el hecho de que las estimaciones puntuales de FIA, VAN y RM resultaran iguales, tanto en la densidad como en el volumen, incluso cuando los conglomerados son incompletos - 1 y c2- no es sorprendente, ya que al considerar que $\bar{a}=\sum_{s=1}^{m} a_{s} / m \Rightarrow \bar{a} m=\sum_{s=1}^{m} a_{s}$ se puede demostrar que los estimadores RM, FIA y VAN son iguales a $y_{h a}=\sum_{s=1}^{m} y_{s} / \bar{a} m$, incluso si los conglomerados son de tamaños diferentes.

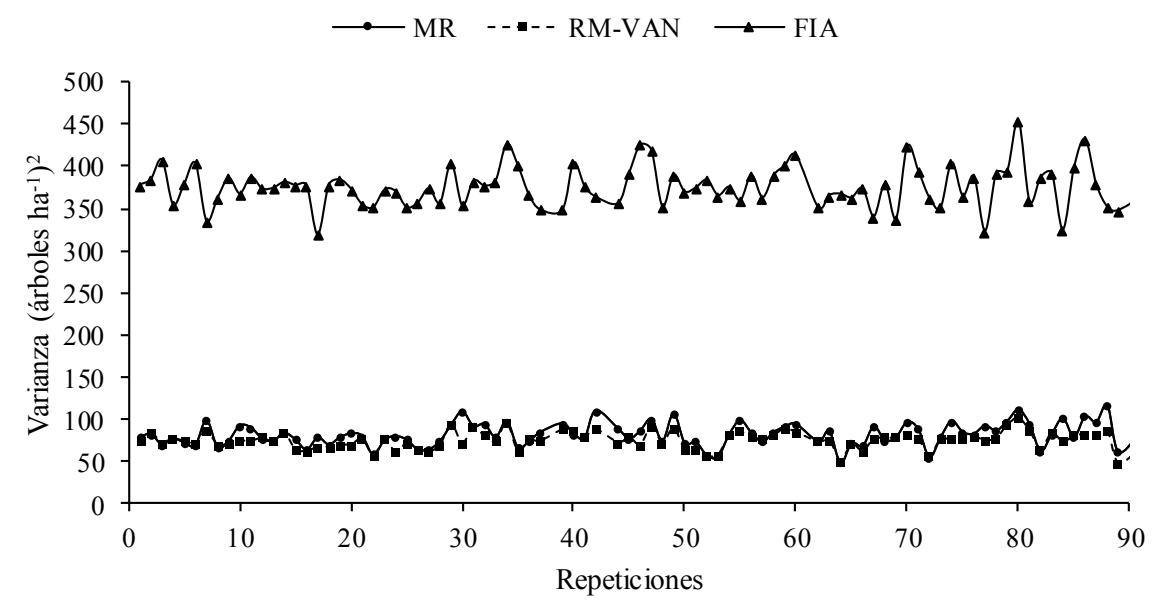

Figura 11. Varianza estimada (árboles ha $\left.{ }^{-1}\right)^{2}$ de cuatro estimadores estudiados en la condición c1. FIA: Forest Inventory and Analysis, VAN: Van Deusen, MR: Media de Razones, RM: Razón de Medias.

Estimated variance (trees $\left.\mathrm{ha}^{-1}\right)^{2}$ of four estimators studied in condition c1. FIA: Forest Inventory and Analysis, VAN: Van Deusen, MR: Mean of ratio, RM: Ratio of means.

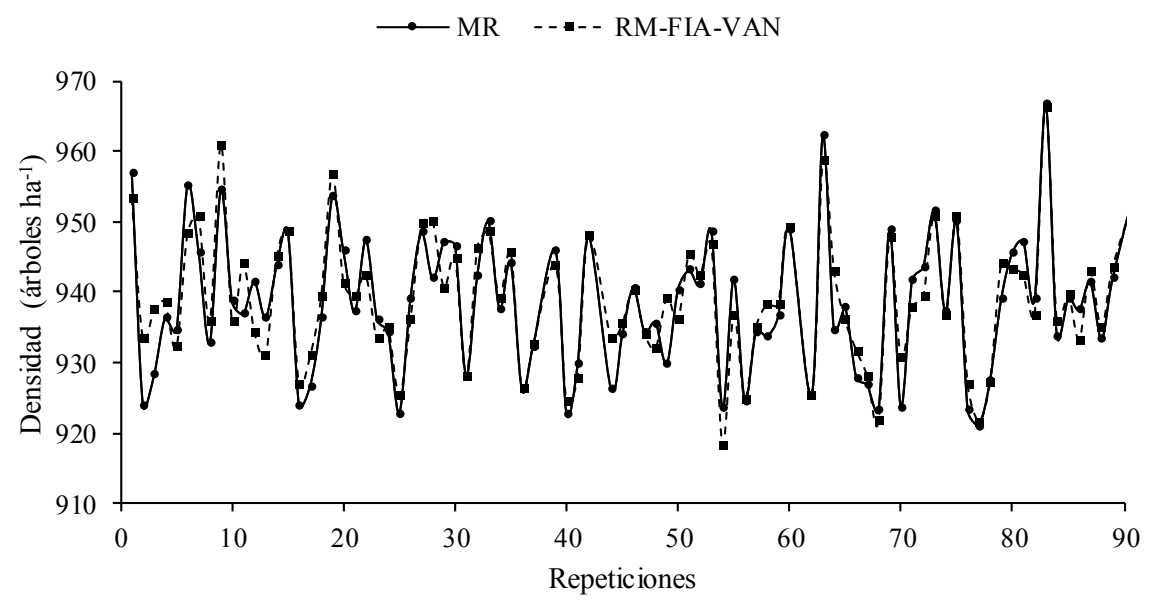

Figura 12. Densidad estimada (árboles ha $\mathrm{a}^{-1}$ ) mediante cuatro estimadores estudiados en la condición c2. FIA: Forest Inventory and Analysis, VAN: Van Deusen, MR: Media de Razones, RM: Razón de Medias.

Estimated tree density (trees ha $\mathrm{a}^{-1}$ ) using the four studied estimators on the $\mathrm{c} 2$ condition. FIA: Forest Inventory and Analysis, VAN: Van Deusen, MR: Means of ratio, RM: Ratio of means. 


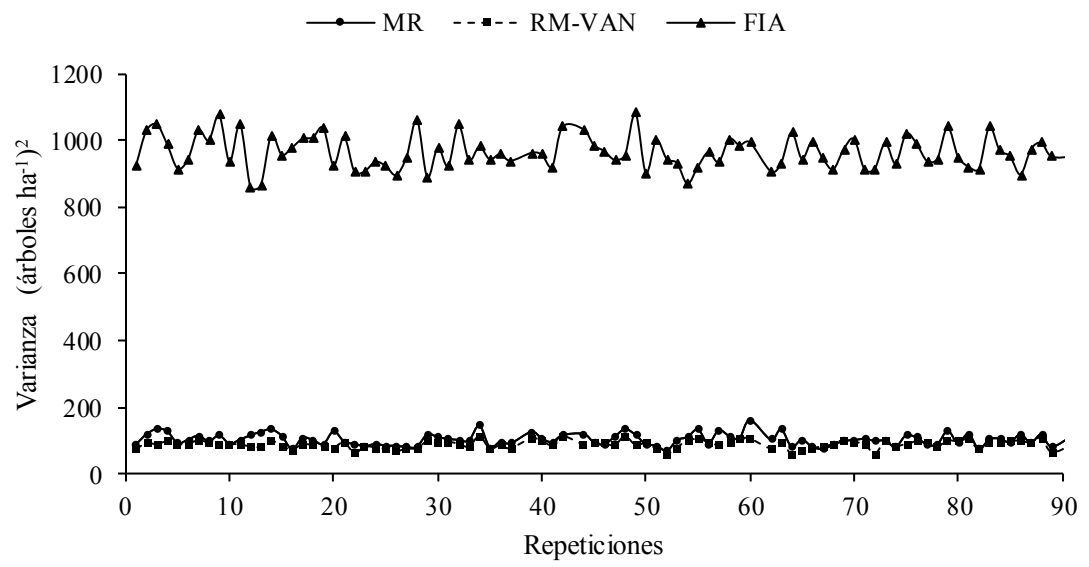

Figura 13. Varianza estimada (árboles ha $\left.{ }^{-1}\right)^{2}$ de cuatro estimadores estudiados en la condición c2. FIA: Forest Inventory and Analysis, VAN: Van Deusen, MR: Media de Razones, RM: Razón de Medias.

Estimated variance $\left(\text { trees } \mathrm{ha}^{-1}\right)^{2}$ on the four studied estimators on the $\mathrm{c} 2$ condition. FIA: Forest Inventory and Analysis, VAN: Van Deusen, MR: Means of ratio, RM: Ratio of means.

Los sesgos de las varianzas relativos en $\mathrm{c} 0 \mathrm{y} \mathrm{c} 1 \mathrm{son}$ congruentes con los reportados por Van Deusen (2004, 2005a). Este autor, al comparar FIA y VAN obtuvo un sesgo relativo de la varianza de FIA de casi 2 cuando analiza una proporción promedio de parcela de 0,75 , mientras que el estimador VAN siempre se comporta insesgado. Estos resultados se deben a que la varianza estimada de FIA se basa en la noción de que cada medición de la parcela se divide por la proporción promedio de la parcela que pertenece a la población forestal de interés. Por lo tanto, el hecho de que esta varianza trabaje pobremente cuando las parcelas tienen proporciones que difieren ampliamente entre conglomerados no es sorprendente.

Los resultados obtenidos por Van Deusen (2005a) indican que las varianzas relativas del estimador MR y VAN también resultaron similares (menores del 0,11), es decir, no hay ganancia significativa en precisión al considerar que la varianza del error es una función de la proporción al cuadrado, por esta razón, el autor no recomienda el uso del estimador media de razones; aunque este tiene la ventaja de proporcionar de manera directa una valor por unidad de muestreo, la cual es información de gran valía cuando es deseable representar espacialmente la variable de interés.

Entonces, resulta evidente que los estimadores MR, RM y VAN pueden tener aplicación general; se desempeñan bien -estimación puntual y por intervalo- cuando en los conglomerados se evalúan las cuatro subparcelas o solo algunas de ellas, por el contrario, la estimación por intervalo del estimador FIA en conglomerados incompletos no es recomendable.

Las varianzas de los estimadores RM y VAN conducen a los mismos resultados porque el factor que relaciona ambas fue de 0,999 y de 0,998 en la c1 y c2, respectivamente. Estos resultados son lógicos al considerar que cuando $\bar{a}=(1 / 2) a_{0}$ y el factor es de 0,9473 (Van Deusen 2005b).

El estimador VAN, además de poder ser aplicado en trabajos de gran escala (INFyS y FIA) también puede usarse en estudios forestales de nivel local, donde las parcelas de muestreo de área fija traslapan el límite de la propiedad. El requerimiento en este caso es el área de las parcelas que pertenece a la propiedad que se requiere evaluar. El supuesto más importante del estimador VAN es que la cantidad esperada del atributo de interés en una parcela parcial sea proporcional al área que pertenece a la población forestal o propiedad objeto de estudio. Van Deusen y Roesch (2009) muestran que el error estándar del estimador VAN se reduce hasta en un $15 \%$ cuando se incluyen parcelas mapeadas que traslapan el límite en comparación que si fueran excluidas. Además de ser un estimador atractivo desde el punto de vista teóricopráctico, el estimador VAN puede aplicarse fácilmente en muestreo aleatorio estratificado (Van Deusen 2005b). En este caso, la media por hectárea de cada estrato se obtiene mediante la expresión del estimador VAN y la varianza estimada de la media por hectárea de cada estrato se obtiene mediante la expresión de la varianza del estimador VAN.

Los estimadores RM y VAN conducen a los mismos resultados, aun en niveles de completitud parcial del conglomerado. De hecho, el estimador VAN es el estimador de razón convencional, con la ventaja de que la varianza estimada considera el área de la parcela efectivamente muestreada en campo. Por este motivo, además de aplicarse para la estimación de atributos agregados tales como el volumen, densidad, biomasa o área basal por hectárea, pueden también emplearse para la estimación de proporciones de alguna característica del arbolado o para la estimación de atributos a nivel de árbol (Smelko y Merganic 2008, Bechtold y Randolph 2018) o incluso a nivel de rodal (Zarnoch y Bechtold 2000). 
A pesar de que el tamaño de muestra utilizado en el presente estudio puede considerarse pequeño -81 conglomerados $^{-}$, se observa que los estimadores evaluados son insesgados independientemente del grado de completitud simulado. Estos resultados son congruentes con los reportados por Van Deusen y Roesch (2009) quienes, mediante simulaciones repetidas 10.000 veces y 1.000 muestras seleccionadas de cada repetición, para un escenario del $80 \%$ de parcelas completas, concluyen que el estimador VAN es insesgado sin importar la exactitud con la que se mapea la parcela.

Takahashi y Tsubaki (2017) consideran que para estimar la media de una variable heterocedástica distribuida log normal no es conveniente aplicar transformaciones logarítmicas, entonces analizan el estimador de mínimos cuadrados generalizados, en cuya expresión la variable auxiliar presenta como exponente un parámetro teta. Al considerar como valores de este a 0,5 y 1 , derivaron respectivamente, el estimador de razón de medias y media de razones. A través de simulaciones de grandes bases de datos de una variable distribuida log-normal, los autores compararon el comportamiento de estos dos estimadores tomando como criterio la raíz del cuadrado medio del error relativo, y concluyeron que para valores cercanos a 0,5 el estimador con mejor desempeño fue el de razón, mientras que para valores alrededor de 1 el estimador de media de razones logró ser el más apropiado. En cualquier caso, y para los 31 valores de teta evaluados, la raíz del cuadrado medio del error relativo no superó el 0,020. Así, bajo 1.000 ensayos del análisis, ambos estimadores fueron insesgados. Luego entonces, y a la luz de los resultados que se logran en la presente investigación, los estimadores RM y MR se consideran apropiados para la estimación de parámetros forestales agregados.

Si bien es cierto que los hallazgos del presente estudio se derivan de una población simulada, resulta interesante observar que son congruentes con aquellos obtenidos desde el punto de vista práctico. Velasco et al. (2016), al comparar estadísticamente los estimadores RM, MR y FIA con datos provenientes de selva mediana subperennifolia y subcaducifolia del sureste de México, encontraron que aún para tamaños de muestra de 22 observaciones no existen diferencias significativas entre los tres estimadores y que el estimador de razón fue ligeramente más preciso. Con 22 observaciones el estimador de razón logró un error de muestreo alrededor del $30 \%$, mientras que con 206 observaciones el error de muestreo fue $8,5 \%$.

Por sus bondades estadísticas, los estimadores que emplean variables auxiliares diferentes del área de la parcela son preferidos en la evaluación de recursos forestales. Fierros et al. (2018) estudiaron el comportamiento del estimador de razón y regresión -bajo muestreo estratificado- para estimar el volumen maderable en plantaciones de pino en México usando como variable auxiliar el área basal. Ellos encontraron que, si bien las estimaciones puntuales resultaron similares, la precisión fue de 1,35\% para el estimador de regresión y de 2,31 \% para el estimador de razón al usar 44 parcelas de muestreo.
Los estimadores comparados en esta investigación se aplican bajo el enfoque de conglomerados incompletos, que no consideran el registro de información dasométrica en las cuatro subparcelas. No obstante, las propiedades de los estimadores FIA, VAN y RM han sido estudiados por Van Deusen $(2004,2005 a, 2009)$ bajo el enfoque de parcelas mapeadas, lo cual significa cuantificar el área de la parcela o unidad de muestreo que corresponde a la población de interés. En uno u otro caso, se justifica el modelo que considera a la varianza del error proporcional al área relativa efectivamente muestreada en campo, y que cualquier otro supuesto no necesariamente implica ganancia significativa en precisión.

Aun cuando los estimadores comparados en esta investigación se derivan a partir de modelos, se considera que la inferencia se realiza en el contexto de inferencia asistida por modelos, en este sentido los cuatro modelos (FIA, VAN, MR y RM) únicamente sirven como una herramienta para encontrar una estimación apropiada de la media. La eficiencia de los estimadores evaluados depende de la bondad de ajuste del modelo. Sin embargo, las propiedades básicas de tales estimadores (insesgadez o validez de las fórmulas de la varianza) no depende sí el modelo se mantiene o no (Särndal et al. 1992, Mandallaz 2008). Además, las cantidades poblacionales se consideran fijas en lugar de variables aleatorias. Cuando el total de la población (o la media) se considera variable aleatoria, el paradigma de inferencia se conoce como inferencia basada en modelos, y en tal caso, el total poblacional (o la media) aleatorio puede ser predicho o su valor esperado puede ser estimado (Ståhl et al. 2016, Saarela et al. 2016).

\section{CONCLUSIONES}

En la condición de completitud total, los cuatro métodos estudiados conducen a las mismas estimaciones y varianzas, de volumen y número de árboles por hectárea. Además, las estimaciones por hectárea y las varianzas estimadas son insesgadas independientemente del estimador. En esta situación, las fórmulas se reducen a los estimadores del muestreo aleatorio simple.

En cada una de las tres condiciones estudiadas, las estimaciones de volumen y número de árboles por hectárea mediante FIA, VAN, MR y RM son similares y prácticamente insesgadas.

A medida que el grado de completitud de los conglomerados es menor, tanto para volumen como para densidad, las varianzas estimadas de VAN, MR y RM son insesgadas, mientras que la varianza estimada de FIA se vuelve fuertemente sesgada.

\section{AGRADECIMIENTOS}

Se extiende un agradecimiento fraterno la Comisión Nacional Forestal de México por haber proporcionado la base de datos del INFyS correspondiente a selva mediana sub-perennifolia de Quintana Roo, México del 2010. 


\section{REFERENCIAS}

Álvarez IA, G Del Nero, H Sundfeld, AM Liner, HT Zarate. 2005. Comparison of two sampling methods for estimating urban tree density. Journal of Arboriculture 31(5): 209-214.

Bechtold WA, KC Randolph. 2018. FIA Crown Analysis Guide. Version 2.0. Washington, USA. United States Department of Agriculture. $37 \mathrm{p}$

Cochran WG. 1993. Técnicas de Muestreo. México, México. Compañía Editorial Continental. 513 p.

CONAFOR (Comisión Nacional Forestal, MX). 2012. Manual y procedimientos para el muestreo de campo: Re-muestreo 2012. Zapopan, Jalisco, México. SEMARNAT. 136 p.

CONAFOR (Comisión Nacional Forestal, MX). 2018. Inventario Nacional Forestal y de Suelos: Informe de Resultados 20092014. Zapopan, Jalisco, México. CONAFOR. 199 p.

Condes S, J Martínez-Millán. 1998. Comparación entre los índices de distribución espacial de árboles más usados en el ámbito forestal. Investigación Agraria: Sistemas y Recursos Forestales $7(1$ y 2$): 173-187$.

ESRI, 2008. ArcGis $9 \circledR$. Geoprocessing Commands Quick Reference Guide. New York, USA. 220 p.

Fierros R, HM de los Santos, AM Fierros, F Cruz, L Martínez, E Velasco, G Quiñónez. 2018. Estimadores de muestreo para inventario de plantaciones de Pinus chiapensis (Martínez) Andresen. Revista Mexicana de Ciencias Forestales 9(47): 131-150. DOI: https://doi.org/10.29298/rmcf.v9i47.155

Gregoire TG, HT Valentine. 2008. Sampling Strategies for Natural Resources and the Environment. New York, USA. Chapman \& Hall/CRC. 474 p.

Lohr S. 2010. Sampling: Desing and Analysis. Second Edition. Boston, USA. Brooks/Cole. 596 p.

Mandallaz D. 2008. Sampling Techniques for Forest Inventories. New York, USA. Chapman and Hall/CRC. 256 p.

Rencher AC, G Bruce. 2008. Linear Models in Statistics. Second Edition. New Jersey, USA. Wiley-Interscience. 672 p.

Saarela S, S Holm, A Grafström, S Schnell, E Næsset, TG Gregoire, RF Nelson, G Ståhl. 2016. Hierarchical model-based inference for forest inventory utilizing three sources of information. Annals of Forest Science 73: 895-910. Consultado 21 mar. 2020. Disponible en https://www.researchgate.net/ publication $/ 308984516$

SAS Institute Inc. 2009. SAS/STAT ${ }^{\circledR}$ 9.2. User's Guide, Second Edition. North Carolina, USA. SAS Institute Inc. 7886 p.

Särndal CE, B Swensson, J Wretman. 1992. Model Assisted Survey Sampling. New York, USA. Springer. 694 p.

Scott CT, WA Bechtold, GA Reams, WD Smith, JA Westfall, MH Hansen, GG Moisen. 2005. Sample-based estimators used by the Forest Inventory and Analysis national information management system. In Bechtold WA, PL Patterson eds. The enhanced Forest Inventory and Analysis program-national sampling design and estimation procedures. General Technical Report SRS-80. Southern Research Station, Asheville, North Carolina. USA. Forest Service. United States Department of Agriculture. p. 43-67.
Scheaffer R, W Mendenhall, R Lyman, KG Gerow. 2012. Elementary Survey Sampling. Seventh Edition. Boston, USA. Brooks/Cole. 436 p.

Smelko S, J Merganic. 2008. Some methodological aspects of the National Forest Inventory and Monitoring in Slovakia. Journal of Forest Science 54(10): 476-483.

Stamatellos G, G Panourgias. 2005. Simulating spatial distributions of forest trees by using data from fixed area plots. Forestry 78(3): 305-312. DOI: https://doi.org/10.1093/forestry/cpi028

Ståhl G, S Saarela, S Schnell, S Holm, J Breidenbach, SP Healey, PL Patterson, S Magnussen, E Næsset, RE McRoberts, TG Gregoire. 2016. Use of models in large-area forest surveys: comparing model-assisted, model-based and hybrid estimation. Forest Ecosystems 3(5): 1-11. Consultado 25 mar. 2020. Disponible en https://forestecosyst.springeropen.com/articles/10.1186/s40663-016-0064-9

Takahashi M, H. Tsubaki. 2017. Imputing the mean of a heteroskedastic log-normal missing variable: A unified approach to ratio imputation. Statistical Journal of the IAOS 33(3): 763-776. Consultado 10 marzo 2020. Disponible en https:// content.iospress.com/articles/statistical-journal-of-the-iaos/ sji160306

Thompson SK. 2002. Sampling. New York, USA. John Wiley \& Sons. 367 p.

Van Deusen PC. 2004. Forest inventory estimation with mapped plots. Canadian Journal of Forest Research 34: 493-497.

Van Deusen PC. 2005a. Comparing mapped plot estimators. In McRoberts RE, GA Reams, PC Van Deusen, WH McWilliams eds. Proceedings of the Sixth Annual Forest Inventory and Analysis Symposium, 21-24 September 2004. Gen. Tech. Rep. WO-70. Denver, CO, USA. Forest Service. United States Department of Agriculture. p. 93-97.

Van Deusen PC. 2005b. Stratified forest inventory estimation with mapped plots. Canadian Journal of Forest Research 35: 2392-2386.

Van Deusen PC. 2007. Weighted estimators for forest inventory mapped plots. Canadian Journal of Forest Research 37: 1142-1145. DOI: https://doi.org/10.1139/X06-277

Van Deusen PC, FA Roesch. 2009. Application of mapped plots for single-owner forest surveys. Journal of Forestry 107: 414417. https://www.fs.usda.gov/treesearch/pubs/36106

Velasco E, H Ramírez, F Moreno, A de la Rosa. 2005. Estimadores de razón para el inventario nacional forestal de México. Revista Ciencia Forestal en México 28: 23-43.

Velasco E, H de los Santos, H Ramírez, G Rendón. 2016. Comparación de estimadores para volumen maderable en selva mediana del sureste de México. Agrociencia 50: 119-132.

Woodall CW, MC Amacher, WA Bechtold, JW Coulson, S Jovan, CH Perry, KC Randolph, BK Schulz, GC Smith, B Tkacz, S Will-Wolf. 2011. Status and future of the forest health indicators program of the USA. Environmental Monitoring Assessment 177: 419-436.

Zarnoch SJ, WA Bechtold. 2000. Estimating mapped-plot forest attributes with ratios of means. Canadian Journal of Forest Research 30: 688-697. 